\title{
The Importance of Real-World Evidence in Understanding Influenza Vaccine Effectiveness
}

\author{
Pietro Ferrara ${ }^{1,2}$, Lorenzo G. Mantovani ${ }^{2,3}$ \\ Department of Public Health, Experimental and Forensic Medicine, University of Pavia, Pavia, Italy \\ Center for Public Health Research, University of Milan - Bicocca, Monza, Italy \\ 3 Value-based Healthcare Unit, IRCCS MultiMedica, Milan, Italy
}

\section{BACKGROUND}

Seasonal influenza is an acute viral respiratory disease which circulates globally with constantly evolving epidemiology [1]. Influenza viruses can infect up to $20 \%$ of the global population each year, resulting in more than 650,000 annual deaths [2]. Seasonal influenza is therefore a major cause of illness, associated with substantial health and economic burdens, due to its impact on healthcare - with increased medical resource utilization and costs, and on society - with enormous death toll and loss of production [3].

Effective vaccines and timely vaccination programs are crucial strategies for the control of seasonal influenza, the spread and severity of which is unpredictable. Influenza viruses are constantly changing due to different types of mutation in viral genes that result in altered surface glycoproteins (hemagglutinin and neuraminidase), which enables viruses to escape the antigen-specific immunity that is induced by prior infections and/or vaccination [1]. Changes in antigenic sites result from either common point mutations during viral replication, which cause small changes in surface proteins (antigenic drift), or from less frequent significant genetic reassortment and the consequent emergence of novel virus subtypes or clades (antigenic shift) [4]. Antigenic evolution and regional variations in environmental strain / clade dominance require that the antigen composition of seasonal influenza vaccines is changed annually $[1,4]$.

Annual vaccination is the best way to prevent influenza disease in people aged 6 months and older [3]. Influenza vaccines are proven to be economically favorable in specific populations including older adults, people with chronic medical problems, pregnant women, and children - from both healthcare and societal perspectives [3]. Therefore, the appropriate evaluation of influenza vaccines based on epidemiological and economic data is of the utmost importance for accurately informing policymakers and allocating resources for seasonal influenza vaccination programs $[2,3]$.

\section{RANDOMIZED CONTROLLED TRIAL (RCT) \\ VS. REAL-WORDL DATA (RWD)}

The evidence in support of the safety, efficacy, and effectiveness of influenza vaccines is based on RCTs and observational studies conducted in real-world conditions [5]. The first are key research tools in studying vaccine candidates, addressing questions on efficacy and incidence of adverse events [6], and measuring influenza vaccine efficacy under specific conditions designed to minimize bias that could invalid experimental results [5]. On the other hand, real-world data (RWD) routinely collected during vaccination campaigns, can assess how well vaccines work in real life conditions, as well as through different seasons and settings, providing actionable metrics towards the continuous monitoring of effectiveness and post-marketing safety surveillance [5].

Influenza vaccines are a paradigm of how real-world evidence (RWE) can offer a complementary wider view of their impact. Due to the constant accumulation of antigenic mutations, influenza viruses change from one year to another, with evolving effects on person-

Corresponding author Pietro Ferrara p.ferrara5@campus.unimib.it

Received: 21 February 2022 Accepted: 23 February 2022 Published: 3 March 2022 
to-person transmission and the severity of disease $[1,2]$. The relationship between efficacy estimates from RCT and vaccines lies at the crossing of evolutionary biology and public health [7,8]. Indeed, RCT estimates refer to a definite vaccine and year, also being measured under the optimal conditions imposed by study design. Due to the high variability between seasonal influenza outbreaks, efficacy results from a given RCT cannot strictly be compared with the results from similar studies conducted in other influenza seasons. This could be due to different annual predominant strains which could impact the severity of the season, and to mismatch between the viruses included in the influenza vaccine and the actual circulating epidemic strains [5,9]. Thus, while RCTs remain the leading design to estimate the protective benefits of influenza vaccines, particularly at the pre-licensure stage, RWE analyses in the post-marketing phase can assess influenza vaccine effectiveness across sequential influenza seasons. This accurately reflects the real extent to which vaccines reduce burden of disease at the population-level over time, also accounting for variations in the timing of disease and vaccination, providing applicable data on possible cross-protection against non-matching circulating viral strains [5,10,11]. Additionally, compared with RCTs, RWD generate estimates using relevant patient-oriented outcomes - namely numbers of cases, medical visits, hospitalizations, or deaths prevented by vaccination - the calculation of which reflects real-world health issues and demonstrates whether interventions provide benefits to patients and improve public health [8,11-13].

Notably, one of the main critical concerns about the evidence generated in vaccine RCTs relates to study population [14]. High-risk groups for influenza-associated complications (such as children younger than 5 years old), individuals with specific chronic conditions, those receiving polytherapy, and pregnant and lactating women are usually excluded from influenza vaccine RCTs. Therefore, the integration of RWE is essential to fulfil the need for reliable and complete evidence demonstrating the effectiveness and benefit-risk profile of vaccination, for both the general population and specific subgroups [14,15].

Vaccine RWE studies assess more varied and larger recipient populations, in different locations, with different levels of risk of infection, and different diseases outcomes. Unlike RCTs, RWE studies are also able to capture the direct and indirect effects of vaccination (including infectiousness and susceptibility, and their duration), as well as other vaccinationrelated aspects - namely vaccination schedules and costs, and population compliance and hesitancy - that significantly change the vaccination coverage rate and the dynamics of transmission between vaccinated and non-vaccinated / naïve individuals [11].

Global mass vaccination to combat the coronavirus (COVID-19) pandemic is a current example of how data derived from RWE analyses have been pivotal in monitoring the contribution of vaccines to pandemic control [16-18]. For instance, RWD routinely collected during national vaccination campaigns have been key to tailoring the most appropriate populationlevel interventions in order to ensure protection against COVID-19, by describing the dynamics of vaccine immunogenicity and effectiveness, targeting the most vulnerable groups, identifying timing for boosters, and solving safety concerns $[17,18]$.

Under the right conditions, RWE provides valid support for health decisions by virtue of data relating to patient health status that can be gathered and integrated from a variety of sources (e.g., clinical health records, disease registries, patient-generated data, etc.) [19]. On this point, it is important to acknowledge some considerations for quality checking RWE in the context of influenza vaccine effectiveness. While evaluating quality of evidence is particularly important for informing health decisions, appropriate and cautious selection of quality assessment tools and strategies is needed in this field [20]. For instance, some frameworks for grading quality of evidence and making recommendations are unlikely to be adequate for understanding the value and reliability of RWE regarding influenza vaccine effectiveness. In the light of the aforementioned constantly evolving viral antigenic variability and influenza epidemiology, the wider view offered by RWE on response to seasonal immunization is likely to be undermined by common approaches (such as the Grading of Recommendations Assessment, Development and Evaluation-GRADE [21]) that tend to prioritize evidence from RCTs, which cannot be considered an extensive metric to support vaccination policy, as discussed above.

Conversely, assessment of RWE in public health interventions should be conducted in the context of a complete overview of the research question and fitness for purpose [20]. While availability of and access to appropriate RWD sources are increasing, the use of a specific tool to assess influenza vaccine effectiveness is required to ensure that RWE is relevant in informing stakeholders and influencing public health policy. 


\section{CONCLUSIONS}

In a nutshell, the potential difference in estimates of vaccine efficacy between RCTs and RWE is an interesting field of investigation. Making comparisons across vaccine studies is complex, with numerous factors and limitations that can potentially affect the validity and reproducibility of results. As regards influenza vaccines, while RCTs designed upon rigorous standards of quality remain important to demonstrate the protective benefits of vaccination, in terms of both safety and efficacy, RWD is a key contributor, able to fill evidence gaps in the field of influenza vaccinology, particularly in terms of the richness and granularity of available data, the advancement of RWE methods, and support for strategies to implement the most appropriate vaccination policies during seasonal influenza outbreaks.

\section{Funding}

This editorial was unconditionally funded by Seqirus Srl.

\section{Conflict of interest}

PF has no conflict of interest to disclose regarding this work

LGM reports support from the Italian Ministry of Health Ricerca Corrente-IRCCS MultiMedica during the conduction of the study; grants and personal fees from Bayer AG, Daiichi Sankyo, Pfizer, and Boehringer Ingelheim outside the submitted work.

\section{REFERENCES}

1. Petrova VN, Russell CA. The evolution of seasonal influenza viruses. Nat Rev Microbiol 2018; 16: 47-60; https://doi.org/10.1038/nrmicro.2017.118

2. World Health Organization - Regional Office for Europe. Influenza - estimating burden of disease. Available at: https://www.euro.who.int/en/health-topics/communicable-diseases/ influenza/seasonal-influenza/burden-of-influenza (last accessed on January 2022)

3. D'Angiolella LS, Lafranconi A, Cortesi PA, et al. Costs and effectiveness of influenza vaccination: a systematic review. Ann Ist Super Sanita 2018; 54: 49-57; https://doi.org/10.4415/ ANN_18_01_10

4. Treanor JJ. Influenza (Including Avian Influenza and Swine Influenza). In: Bennett JE, Dolin R, Blaser MJ. Mandell, Douglas, and Bennett's Principles and Practice of Infectious Diseases (Eighth Edition). W.B. Saunders, 2015: Pages 2000-2024.e6; https://doi.org/10.1016/ B978-1-4557-4801-3.00167-3

5. US Centers for Disease Control and Prevention - National Center for Immunization and Respiratory Diseases (NCIRD). How Flu Vaccine Effectiveness and Efficacy are Measured. Available at: https://www.cdc.gov/flu/vaccines-work/effectivenessqa.htm (last accessed on January 2022)

6. Balasingam S, Wilder-Smith A. Randomized controlled trials for influenza drugs and vaccines: a review of controlled human infection studies. Int J Infect Dis 2016; 49: 18-29; https://doi.org/10.1016/j.ijid.2016.05.013

7. Boni MF. Vaccination and antigenic drift in influenza. Vaccine 2008; 26: C8-14; https:// doi.org/10.1016/j.vaccine.2008.04.011

8. US Centers for Disease Control and Prevention - National Center for Immunization and Respiratory Diseases (NCIRD). Influenza Vaccines for Older Adults. Advisory Committee on Immunization Practices (ACIP) January 12, 2022

9. Jayasundara K, Soobiah C, Thommes E, et al. Natural attack rate of influenza in unvaccinated children and adults: a meta-regression analysis. BMC Infectious Diseases 2014; 14: 670; https://doi.org/10.1186/s12879-014-0670-5

10. Tokars JI, Rolfes MA, Foppa IM, et al.. An evaluation and update of methods for estimating the number of influenza cases averted by vaccination in the United States. Vaccine 2018; 36: 7331-7; https://doi.org/10.1016/j.vaccine.2018.10.026 
11. Flannery B, Fry AM. Comparing Influenza Vaccine Types: The Path Toward Improved Influenza Vaccine Strategies. J Infect Dis 2019; 220: 1237-9; https://doi.org/10.1093/ infdis/jiy 717

12. Halloran ME, Struchiner CJ, Longini IM. Study designs for evaluating different efficacy and effectiveness aspects of vaccines. Am J Epidemiol 1997; 146: 789-803; https://doi. org/10.1093/oxfordjournals.aje.a009196

13. Heneghan C, Goldacre B, Mahtani KR. Why clinical trial outcomes fail to translate into benefits for patients. Trials 2017; 18: 122; https://doi.org/10.1186/s13063-017-1870-2

14. Martini N, Trifirò G, Capuano A, et al. Expert opinion on Real-World Evidence (RWE) in drug development and usage. Pharmadvances 2020; 2: 41-50; https:/doi.org/10.36118/ pharmadvances.02.2020.01

15. Uyeki TM. High-risk Groups for Influenza Complications. JAMA 2020; 324: 2334; https:// doi.org/10.1001/jama.2020.21869

16. Ponticelli D, Madotto F, Conti S, et al. Response to BNT162b2 mRNA COVID-19 vaccine among healthcare workers in Italy: a 3-month follow-up. Intern Emerg Med 2021: 1-6; https://doi.org/10.1007/s11739-021-02857-y

17. Ponticelli D, Antonazzo IC, Caci G, et al. Dynamics of antibody response to BNT162b2 mRNA COVID-19 vaccine after 6 months. J Travel Med 2021; 28: taab173; https://doi. org/10.1093/jtm/taab173

18. Ferrara P, Antonazzo IC, Polosa R. Response to BNT162b2 mRNA COVID-19 vaccine among healthcare workers in Italy: A 3-month follow-up-Reply. Intern Emerg Med 2022; 17: 313-14; https://doi.org/10.1007/s11739-021-02911-9

19. US Food and Drug Administration. Real-World Evidence. FDA: 2022. Available at: https:// www.fda.gov/science-research/science-and-research-special-topics/real-world-evidence (last accessed on January 2022)

20. Reynolds MW, Bourke A, Dreyer AN. Considerations when evaluating real-world data quality in the context of fitness for purpose. Pharmacoepidemiol Drug Saf 2020; 29: 13168; https://doi.org/10.1002/pds.5010

21. Schünemann, Brożek J, Guyatt G, et al. GRADE Handbook. Handbook for grading the quality of evidence and the strength of recommendations using the GRADE approach. Updated October 2013. Available at: https://gdt.gradepro.org/app/handbook/handbook. html (last accessed on January 2022) 